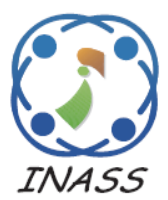

\title{
Adaptive ABC Algorithm Based PTS Scheme for PAPR Reduction in MIMO- OFDM
}

\author{
Vijayalakshmi Maddala ${ }^{1^{*}}$ \\ Ramalinga Reddy Katta ${ }^{1}$ \\ ${ }^{1}$ Gunampalli Narayanamma Institute of Technology and Science, Hyderabad, Telangana, India \\ * Corresponding author's Email: vijayalakshmim8120@gmail.com
}

\begin{abstract}
The OFDM signals which have a broad peak-to-average power ratio is a basic prerequisite to be settled. The Orthogonal Frequency Division Multiplexing (OFDM) traces the Partial transmit sequences (PTS) which is one among the appealing procedures to diminish the peak-to-average power ratio (PAPR). An issue of modern computational multifaceted nature happens in The PTS scheme when prevalent quantities of sub blocks were consumed. In this article, another PTS scheme has been industrialized so as to decline the PAPR by picking the ideal stage factor esteem by means a versatile ABC optimization process. At the point when associated with MIMOOFDM structures with a broad assortment of phase factors ABC based PTS calculation achieves about elite after simulation. It can essentially diminish the computational intricacy for bigger PTS subblocks and offers lower PAPR in the meantime. The anticipated philosophy is connected in the working stage of MATLAB and the utilitarian solutions were incited and researched.
\end{abstract}

Keywords: OFDM, PTS, PAPR, ABC optimization process.

\section{Introduction}

OFDM is an alluring air-connect expertise for fast information transmission wireless communication because of its inborn durability to the frequency selective fading channel and high spectral efficiency. Thusly, OFDM has been used in various wireless benchmarks, to be specific, Digital Audio Broadcasting (DAB), Terrestrial Digital Video Broadcasting (DVB-T), IEEE 802.11a standard for Wireless Local Area Networks (WLAN) and the IEEE 802.16a standard [1, 2]. A pivotal burden of the OFDM-based plan is its high peak-to-average power ratio (PAPR) at the transmitter, requiring the use of a linear amplifier that prompts to low power adequacy. For sensible power viability, OFDM flag level ought to be near the non-direct field of the enhancer, going from side to side non-linear modifications and degrading the blunder enactment [3].

Repeated clipping and filtering (RCF) is a trademark system of PAPR lessening techniques which is easy to apply. In any case, in the technique a foreseen PAPR decreasing which have a lot of iterations is mandatory. To diminish the high PAPR of the OFDM, various PAPR lessening frameworks containing the partial transmit sequence (PTS), selective mapping (SLM), and nonlinear companding have been anticipated $[4,5]$. The partial transmit sequence (PTS) is a deception less framework on the premise of joining signal sub blocks that are phase moved by consistent phase elements. The strategy can get sufficient PAPR decrease and side information necessity to be sent at the comparative time frame. However, the exhaustive inquiry multifaceted nature of the ideal phase amalgamation upsurges exponentially with the measure of sub-blocks. Thusly various imperfect PTS approaches have been built up. The iterative flipping algorithm expected for PTS has the computational complexity which is straightly proportional to the quantity of sub blocks. A neighborhood search is anticipated by gradient descent search [6].

By adapting the issue into a relating issue a problematic method is industrialized for minimizing the sum of stage pivoted vectors. The viability of these methodologies upsurges with the quantity of 
stages from the deliberate set. The efficiency of the PTS strategy likewise upsurges if a higher number of blocks are used. The downside is that a superior viability requires an amplified measure of calculation at the transmitter's side furthermore at the receiver side [7, 8]. Another technique to lessen a PAPR, single carrier frequency division multiple access (SCFDMA) was SLM. It produces various OFDM symbol candidates for all data. Since the phases of the subcarriers are pivoted unusually, the worldly waveforms formed by subjecting them to IFFT income has dissimilar PAPR values. A receiver needs to recognize the pivot used to produce the transmitted signal for demodulation. The dissemination of the revolution information eats up radio sources to some degree and this happens with each OFDM image in basic SLM. The computational entanglement appears to be more noteworthy and BER enactment is not appropriate with the assistance of SLM strategy $[9,10]$.

Additionally a problematic stage improvement conspire utilizing versatile $\mathrm{ABC}$ based PTS technique is presented which can productively diminish the PAPR of OFDM signs. The proposed plan can look the better mix of the initial phase factors. Simulation outcomes demonstrate that the versatile $A B C$ based PTS phase optimization plan can accomplish predominant PAPR decrease execution and in the meantime requires far less computational intricacy than the past PTS methods.

This paper is composed as follows. In Section II, we survey distinctive PAPR diminishment strategies. The standards of PTS strategies are portrayed and the versatile $A B C$ based PTS algorithm to seek the better blend of the phase factors is proposed in Section III. In Section IV, the execution of versatile ABC based PTS algorithm and different algorithms for PAPR decrease is assessed by simulation. Conclusions are made in Section V.

\section{Recent Literature Review}

A dissimilar distortion less peak-to-average power ratio (PAPR) discount technique that could be easily executed with no symmetry requirement in direct-detection (DD) optical orthogonal frequency division multiplexing (O-OFDM) structures on the premise of the fast Hartley transform (FHT) was proposed by L. Nadal et al [11]. The capacity of DD O-OFDM plans was confined by the limitations on plan constituents like digital to-analog converter (DAC), the Mach-Zehnder modulator (MZM), analog to-digital converter (ADC), and electrical enhancers. Keeping in mind the end goal to lessen the requirements on these constituents, they correspondingly clip the conveyed signal and execute low complexity (LC) with less distortion. PAPR lessening frameworks which can alleviate at the comparative time frame accomplishes PAPR quantization and clipping noise. Executing LCselective mapping (SLM) with no supplementary change obstruct, the PAPR diminishment was not accomplished in particular. It is actualized with just a single supplementary FHT block by LC-partial transmit sequence (PTS).

A nonlinear impact convinced utilizing the Mach-Zehnder modulator (MZM) and optical selfphase modulation (SPM) in the presence of high peak to-average power ratio (PAPR) was considered hypothetically and is given by $\mathrm{H}$. Chen et al [12]. The direct discovery optical orthogonal frequency division multiplexing (DD-OOFDM) conspire with an electronic pre-bending technique for companding transform (CT) was used to diminish the peak toaverage power ratio (PAPR) of OFDM signals and advance the recipient affectability. It shows that the PAPR diminishment couldn't moderate the nonlinearity of MZM adequately.

An aggregate peak to-average power ratio (PAPR) decrease strategy for excessively trimmed optical orthogonal frequency division multiplexing (ACO-OFDM) plan was given by J. Zhou et al [13]. PAPR-diminishment strategy syndicates discrete Fourier transform (DFT)- spread technique with peak-clipping strategy. For a tradeoff inside PAPR and BER work, the clipping ratio (CR) in peakclipping technique was set to 2.2. Underneath this $\mathrm{CR}$, a $6 \mathrm{~dB}$ of PAPR diminishment could be accomplished by the assembled PAPR-decrease strategy at the likelihood of 103 and BER execution was not really overstated utilizing the clipping deception. At the forward error correction (FEC) restrain, ACO-OFDM with the assembled PAPRdecrease technique had exceptionally poor improvement of set up affectability connected with ACOOFDM.

A sort of invertible subset low-density parity check (IS-LDPC) codes were used to decrease the peak to-average power ratio for orthogonal frequency division multiplexing plans with low complexity. of late, the critical indication in it was that each invertible subset could act naturally adequately altered to create other legitimate code expressions of the LDPC code and this was proposed by S. Shu et al [14]. QC-LDPC (IS-QCLDPC) codes connected two sorts of subset dividing and the advantages of IS-QC-LDPC codes with disparate subset apportioning were thought. Subsequent to declaring that inserted partitioning was more suitable for IS-QC-LDPC codes than the 
block partitioning, edge graph like calculation makes some inadmissible IS-QC-LDPC codes which may increase the peak-to-average power ratio.

A PAPR decrease strategy was depicted by $\mathrm{K}$. Kim [15]. Orthogonal frequency division multiplexing (OFDM) signals have high peak toaverage power ratio (PAPR) that outcome in bending if OFDM flag licenses by means of a nonlinear high power enhancer. A partial transmit sequence (PTS) framework was one among the trademark PAPR diminishment approaches. A cyclic shifted sequences (CSSs) framework was advanced from the PTS framework to propel the PAPR diminishment institution, in which OFDM flag subsequences were consistently moved and joined to deliver substitute OFDM signal series. The shift value (SV) gathers in the CSS framework could be judiciously assigned since they were carefully connected with the PAPR capacity of the CSS framework. The blunders in shift value bring about expanded peak to-average power ratio.

Low-density parity check (LDPC) codes known as invertible subset LDPC (IS-LDPC) code expect for peak to-average power ratio (PAPR) decrease in OFDM designs with low unpredictability and are given by $\mathrm{D}$. Qu et al [16]. An IS-LDPC code has various disjoint invertible subsets, and each invertible subset could act naturally reliantly upturned to deliver other legitimate code expressions of the LDPC code. To idea IS-LDPC codes with great mistake adjusting execution, an adjusted dynamic edge-growth construction algorithm was used. The IS-LDPC codes show poor blunder amending establishment and the PAPR diminishment on the premise of IS-LDPC codes are likewise not effective.

\section{Proposed Methodology}

Multi-antenna transmission and reception (called as MIMO) is broadly inclined as the fundamental aptitude for enabling wireless broadband services, whose across the board achievement will require 10 times advanced spectral viability than existing cell plans and at 10 times low cost per bit. In the various high data rate executions OFDM (orthogonal frequency division multiplexing) technique has been upheld as the standard. The use of parallel OFDM known as MIMO-OFDM can be used in the next generation communication plans, where the PAPR (Peak to Average Power Ratio) rebate is a fundamental outline trouble.

PAPR diminish process relies on dissimilar basics of the outline. There are different constituents that are to be considered before an auxiliary PAPR drop off strategy of the blueprint i.e., complexity, growth in diffuse flag power and enlargement in the bit-mistake rate at the beneficiary, misfortune in information rate.

In the part of scrambling upsets to assemble the subcarriers the Partial Transmit Series is a bending less strategy. The essential thought of PTS approach is that the info data piece is estranged into isolated sub-blocks. The sub-transporters that are diffused by means of the sub blocks are multiplied by measuring estimation of the stage revolution vector expected for those sub pieces.

The stage transformation vector is efficiently chosen to such an extent, to the point that the PAPR esteem is decreased. PTS is exceptionally operative in PAPR diminishing and strong severance utilization. On another perspective momentous computational complexity is essential to look concerning high-dimensional vector space together with imperative transmission of side information (SI) to the receiver is entangled for a practical execution.

Thus, in this article a successful streamlining method is built up inside the PTS plan to show the stage pivot vector with less calculation confusion. The adaptive Artificial Bee Colony (i.e., coordination of $\mathrm{ABC}$ and Cuckoo Search Optimization) algorithm is the improvement procedure acclimated in our anticipated strategy.

\subsection{PAPR in OFDM System}

Take up that the OFDM scheme has ' $K$ ' number of sub carriers in the parallel transmission scheme. The $n^{\text {th }}$ multi faceted vapid symbol in a block of $K$ data symbols can be provided as $Y_{n}(0 \leq n \leq K-1)$.

Besides, the frequency domain information symbol vector with $K$ subcarriers can be transliterated as,

$$
Y_{n}=\left[\begin{array}{lllllll}
Y_{0} & \ldots & Y_{K-1} & \ldots & Y_{K} / 2 & \ldots & Y_{K-1}
\end{array}\right]^{T}
$$

Where, $Y_{n}$ - input data block

At present, N-point inverse fast Fourier transform (IFFT) is tied up to accomplish the time domain OFDM signal for all the sub carriers. The yield of IFFT for $y_{k}$ can be delivered by the underneath demonstration:

$$
y_{k}=\frac{1}{\sqrt{K}} \sum_{n}^{K-1} Y_{n} \exp \left(\frac{i^{2} \Pi n k}{K}\right)
$$

Where $i^{2}=-1$

Similarly, the PAPR of the OFDM signal that is the ratio of the maximum power to the average of 
the power signal is established as,

$$
\text { PAPR Rate }=10 \log \frac{\max _{k}\left\{\left|y_{k}\right|^{2}\right\}}{\exp \left\{\left|y_{k}\right|^{2}\right\}}
$$

Where,

$\exp \{$.$\} - Expected value operator$

Frequently, the PAPR will be regarded as in the name of the harmonizing cumulative distribution function ( $\overline{C D F}$ ). The analogous cumulative distribution enactment can be exploited to evaluate and subordinate the enactment of any PAPR drop off methods.

At this juncture, the $\overline{C D F}$ of the distinct time PAPR is quantified as follows,

$$
\begin{aligned}
& \overline{C D F}=\operatorname{cmpr}_{-} \operatorname{perf}\left(P A P R>P A P R_{0}\right) \\
& \overline{C D F}=1-\left(1-e^{-P A P R_{0}}\right)^{K}
\end{aligned}
$$

\subsection{Proposed Optimal Partial Transmit Sequence (PTS) Technique}

The anticipated Optimal Partial Transmit Sequence technique is made known by the beneath block diagram illustrated in figure 1.

In Partial Transmit Sequence system, the frequency domain vector ' $Y$ ' is separate out into separate ' $R$ ' sub-blocks. The separating of subblocks is indicated as,

$$
Y=\sum_{r=1}^{R} Y^{(r)}
$$

Where,

$$
\begin{gathered}
Y^{(r)}=\left[Y_{0}^{(r)}, Y_{1}^{(r)}, \ldots, Y_{K-1}^{(r)}\right] ; \\
Y_{n}^{(r)}=Y_{n} \text { (or) } 0(1 \leq r \leq R)
\end{gathered}
$$

Cogitate, $a=\left\{a_{r}=e^{i r_{r}}, r=1,2, . ., R\right\}$ be the group of phase factors that are employed to the sub blocks. These days, the above equation paybacks,

$$
Y^{\prime}=\sum_{r=1}^{R} a_{r} Y^{(r)}
$$

Where, $a_{r}=e^{i{ }^{i p r}}, r=1,2, . ., R$.

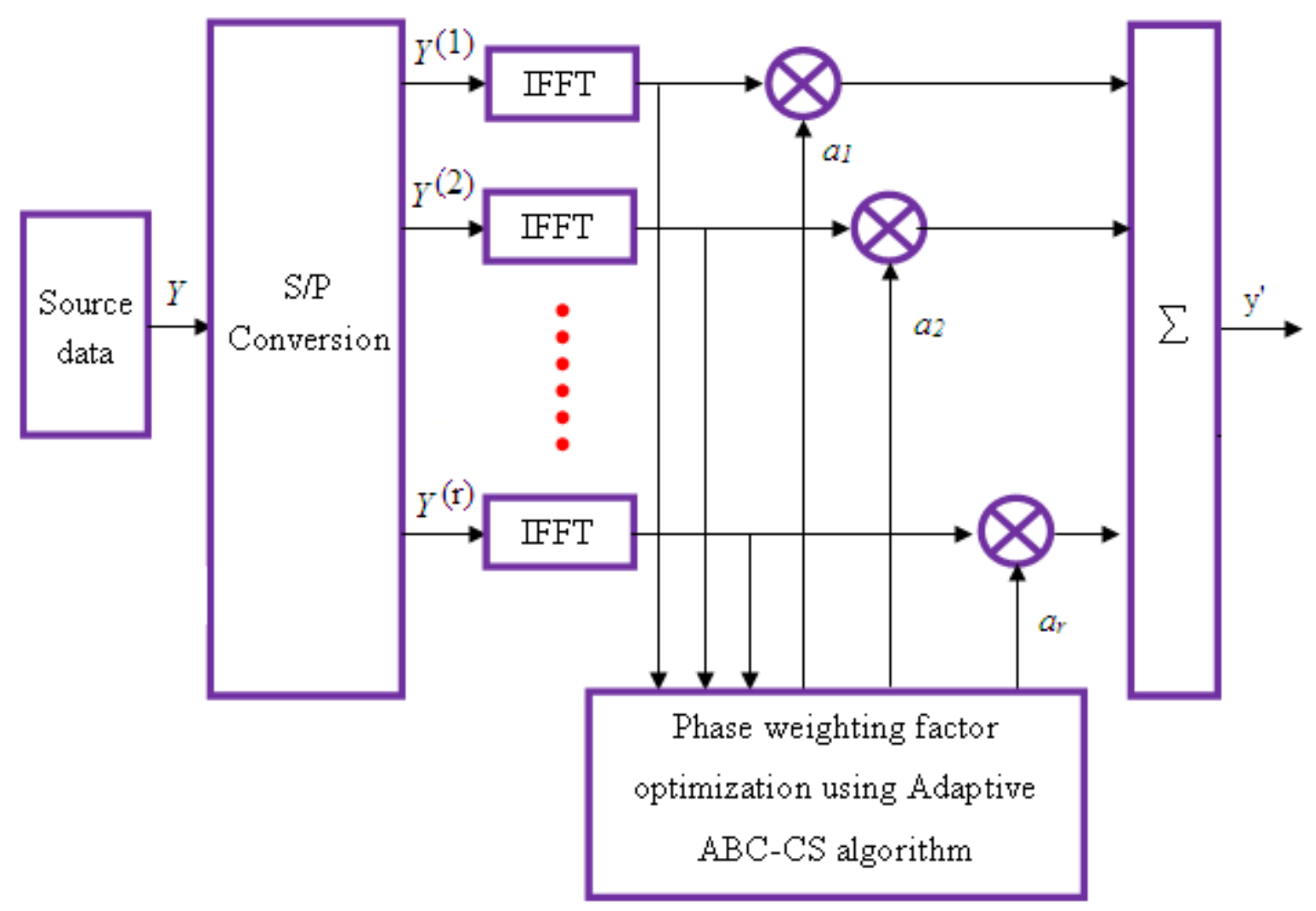

Figure.1 Block Diagram of projected optimal PTS scheme 
On covering the inverse fast Fourier transform (IFFT) and then the linearity property to the above equation, the time domain partial transmit series can be achieved as,

$$
\begin{array}{r}
y^{\prime}=\operatorname{IFFT}\left\{Y^{\prime}\right\} \\
\text { Henceforth, } y^{\prime}=\sum_{r=1}^{R} a_{r} y^{(r)}
\end{array}
$$

In the PTS scheme, one suitable amalgamation from phase factor $\left(a_{r}(r=1,2 \ldots R)\right)$ is to be chosen appropriate to attain the optimal outcome (i.e., lower PAPR).

The amalgamation is presented as follows,

$$
a=\underset{\left(b_{1}, b_{2}, \ldots b_{v}\right.}{\arg \min }\left(\max _{1 \leq k \leq K}\left|\sum_{r=1}^{R} a_{r} Y_{r}\right|^{2}\right)
$$

Where, $\arg \min ($.$) is the judgment performance$ to judge the situation whether the significance is having the least value. The ideal stage figure diminishes the PAPR esteem however the computation of ideal stage element is more multifaceted as it requires large number of calculations. Right now, we are proposing a versatile $A B C$ advancement procedure to upgrade the phase factor esteem.

\subsubsection{Adaptive ABC (ABC-CS)}

In this work, a versatile ABC strategy is introduced to enhance the PTS conspire keeping in mind the end goal to diminish the PAPR (Peak to Average Power Ratio) in the MIMO-OFDM plans. Right now, the Artificial Bee Colony calculation is mongrelized with the Cuckoo Search enhancement calculation to give the ideal stage figure esteem. The $\mathrm{ABC}$ and the CS calculation is exhaustive in the underneath portions.

\section{Artificial Bee Colony Algorithm}

The Artificial Bee Colony calculation was acclimated by Karaboga in 2005 for advancing numerical issues that contain three sets, overwhelmingly:

\section{Employed Bees \\ Onlooker Bees and \\ Scout Bees}

The bee performing search randomly is called as a scout. The bee heading off to the sustenance source remained by it before and imparting its information to others honey bees is perceived as employed bee and the bee attending to the move field is perceived as spectator bee. The spectator bee and scout is otherwise called unemployed bee.

$\mathrm{ABC}$ calculation named the aggregate knowledge looking model in this way includes three crucial modules:

\section{Employed bees}

Unemployed bees and

Food sources.

The utilized and unemployed bees look for the rich sustenance sources round their hive. Similarly, in the part of enhancement structure, the quantity of sustenance assets in $\mathrm{ABC}$ means the number of inhabitants in possible outcomes. The position of a decent sustenance source assigns the position of a superior outcome to the provided advancement issue. The nature of nectar of a nourishment asset describes the wellness of the related outcome.

The sustenance source position implies an outcome to the improvement issue and the nectar measure of a nourishment source consents to the wellness of the reliable outcome in $\mathrm{ABC}$ calculation.

The far reaching stream chart of the versatile $\mathrm{ABC}$ (incorporated ABC-CS) streamlining procedure is indicated with the assistance of the figure 2 . 


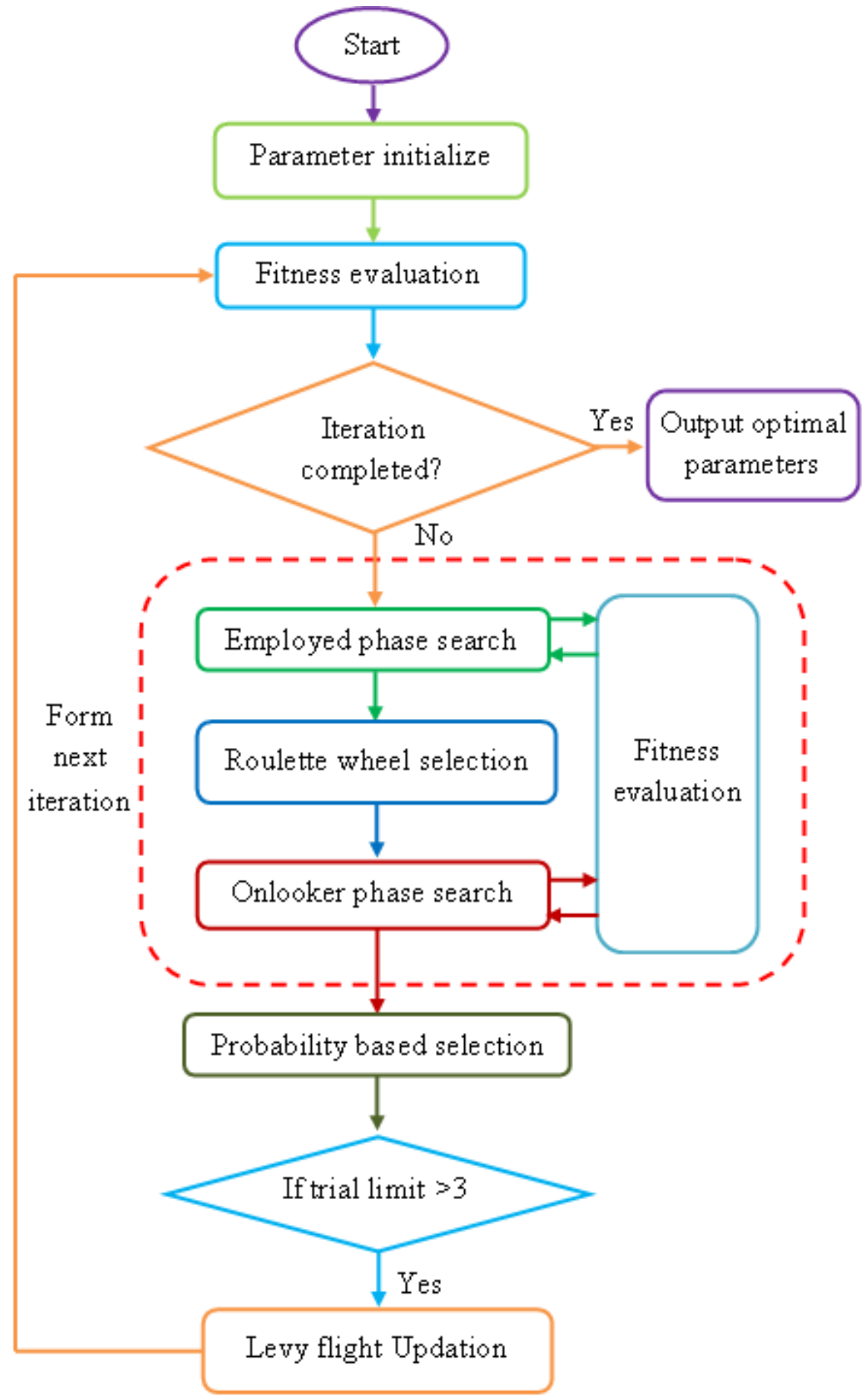

Figure.2 Flowchart of Adaptive ABC optimization process

\section{Cuckoo Search Optimization algorithm}

Cuckoo search algorithm is a metaheuristic calculation set up by Xin-She Yang and Suash Deb in 2009 by motivation from nature. The calculation is on the premise of commit brood parasitism of certain cuckoo species and discretionary strolls known as Lévy flights.

Cuckoo seek calculation rely on the escorting three standard principles.

1. Each cuckoo picks heedlessly a home and lays a solitary egg in the home at once.

2 . The best homes (outcomes) measured utilizing the target execution arranged by the issue is proceeded to the consequent eras.

3. The open home number is settled and the likelihood of discovering (probability) the cuckoo eggs by host flying fowls is in the extent of $[0,1]$. 


\section{Steps in Adaptive Artificial Bee Colony Cuckoo Search Optimization Procedure}

The stages confounded in the hybrid ABC CS streamlining procedure is given by utilizing the succeeding section,

\section{Step 1}

At the principal stage, the $A B C$ creates a randomly distributed primary population of results $(S)$, where $S$ signifies the size of employed bees or onlooker bees.

Each outcome, $t_{n}(n=1,2, \ldots, S)$ is a $M$ dimensional vector. Where $(n=1,2, \ldots, S)$ and $M$ is the number of optimization strictures.

$$
t_{n p}=t_{\min p}+\text { rand }[0,1]\left(t_{\max } p-t_{\min p}\right)(10)
$$

\section{Step 2}

After initialization, the preliminary fitness of the population is evaluated. The fitness can be measured with the help of the underneath equation.

$$
\text { fitness }=\min (\text { PAPR })
$$

At this time, the objective enactment is the least of the PAPR value. The population of the results is then exposed to recurrent cycles like employed bees, the onlooker bees and the scout bees.

\section{Step 3}

For every single employed bee new answers $\left(E_{n p}\right)$ is formed by the outcome pursuit equation.

$$
E_{n p}=t_{n p}+\psi_{n p}\left(t_{n p}-t_{q p}\right)
$$

Where,

$\psi$ - Uniform random number

$p$ - Dimension

$n$-food source

$$
\begin{aligned}
& p €\{1,2, \ldots, S\} \\
& n €\{1,2, \ldots, M\}
\end{aligned}
$$

The outcome satisfying the objective function is nominated and is commanded to the successive phase (onlooker bee phase).

\section{Step 4}

In this phase, the probability values $\left(p r o b_{n b}\right)$ for the outcomes $\left(E_{n p}\right)$ are well thought-out by means of the following eq. (13),

$$
a=\underset{\left(b_{1}, b_{2}, \ldots b_{v}\right)}{\arg \min }\left(\max _{1 \leq k \leq K}\left|\sum_{r=1}^{R} a_{r} Y_{r}\right|^{2}\right)
$$

When a position cannot be enriched further over a prearranged number of cycles, the food source should be unobstructed (i.e., the food source is uninhibited if the same food source is assimilated as the fittest one for the three trial counts).

\section{Step 5}

The out of control outcomes are determined, if exists, and the outcomes are replaced with a novel randomly produced outcome $\left(L_{n p}\right)$ (By means of Lévy Flight Updation).

The Lévy flight is solitary amongst the methodologies that specific animals use to search for food in nature. Additionally, the Lévy flight can be distinct as the random interrupt distributes the step lengths on the basis of the Lévy distribution which has an infinite variance with an infinite mean. follows

The Lévy flight distribution can be quantified as

$$
\text { Levy } z=d^{-\alpha},(1 \leq \alpha \leq 3)
$$

On the basis of the Lévy flight distribution, the outcome can be updated by the subsequent relation,

$$
t_{n p}^{d+1}=t_{n p}^{d}+\chi \oplus \operatorname{Levy}(\alpha)
$$

Where,

$\chi$-parameter to control step size $(\chi>0)$

\section{Step 6}

Commit to memory the finest outcome that is attained thus far.

\section{Step 7}

Repeat the cycle up to the closure disorder is satisfied.

When the termination criteria are prolonged, the optimal value of the phase factor is accomplished. The PTS scheme can be premeditated in the MIMOOFDM scheme to decline the PAPR without deteriorating the spectral effectiveness of the scheme with the optimal phase factor value.

\section{Result and Discussion}

The simulation for the proposed algorithm is accomplished on MATLAB 7.0 version 2014a having 8 GB RAM with 32 bit operating system. Random OFDM symbols are formedto get CCDF. For accurate PAPR The transmitted signal is oversampled using a factor of $L$. 16-QAM modulation with $N$ sub-carriers is exploited and the phase factor $W$ is carefully chosenin our simulation. When larger phase factor, for example, $W=4$ is selecteda better-quality PAPR lessening is accomplished. At this time, $\mathrm{W}$ is changed iteratively 
in $[0,2 \pi]$. Computation time and memory are important to sham higher subblocks with more phase factor amalgamation which is high. PAPR reduction technique identified as Partial Transmit Sequence technique is selected together with unalike optimization systems used for lessening the searching intricacy of phase factor.

\subsection{Performance Calculation}

The enactment assessment of OFDMA and plentiful optimization answers are explicated. The simulation parameters used are SER, BER, PAPR which can be labelled as follows,

\section{BER}

The BER is ratio of error bits to the whole number of bits transmitted all through the time interval i.e.

$$
B E R=(\text { error bits }) /(\text { no.of transmitted bits })(16)
$$

\section{* SNR}

The SNR is is well-defined as the ratio of signal power to the noise power and is uttered in $\mathrm{db}$.

$$
S N R=S p / N p
$$

BER is articulated in the name of SNR. BER is self-possessed by linking the transmitted signal with well-known signal, and compute the error counts over the complete number of bits conveyed.

\section{Peak-to-Average Power Ratio}

In an OFDM conspire a substantial number of independently tempered sub-transporters exists. The pinnacle estimation of the plan can be high as connected with the average of the entire plan. This ratio of the peak to average power esteem is named as Peak-to Average Power Ratio. Coherent expansion of $\mathrm{N}$ signs of same stage yields a pinnacle that is $\mathrm{N}$ times the average signal.

An awesome measure of symbol errors prompts to high noise power, thus it produces ISI in the framework. By collecting the estimation of SNR up to a firm level we can decrease the Symbol Error Rate (SER) furthermore the ISI. In figure 3, SER capacity of OFDM plan is explored with different improvement strategies. In the SER the current Cuckoo Search at iteration 1000 is 10-0.3though by Artificial Bee Colony it is10-0.45 The SER esteem achieved with the assistance of proposed Artificial Bee Colony Cuckoo Search is10-0.42. Thus our proposed ABC-CS conspire outperforms when contrasted with the current $\mathrm{ABC}, \mathrm{CS}$ as far as SER and computational intricacy.

The high pinnacles of the OFDM flag birthplaces a considerable in-band mutilation that hints to griminess in the BER execution. In figure 4, BER capacity of OFDM plan is researched with unique advancement strategies. The BER expected by existing Cuckoo Search at cycle 1000 is 100.41 though by method for Artificial Bee Colony it is 10-0.41. The BER esteem accomplished by proposed Artificial Bee Colony Cuckoo Search is100.4. Accordingly it advances execution of OFDM plan. Existing strategies can diminish PAPR somewhat and more often result in awesome debasement of BER, which makes them unconventional in genuine framework. Our proposed ABC-CS calculation settle this problem via precisely picking the peak esteem and straightening out the periods of the heavenly body focuses, which keeps the BER from falling apart.

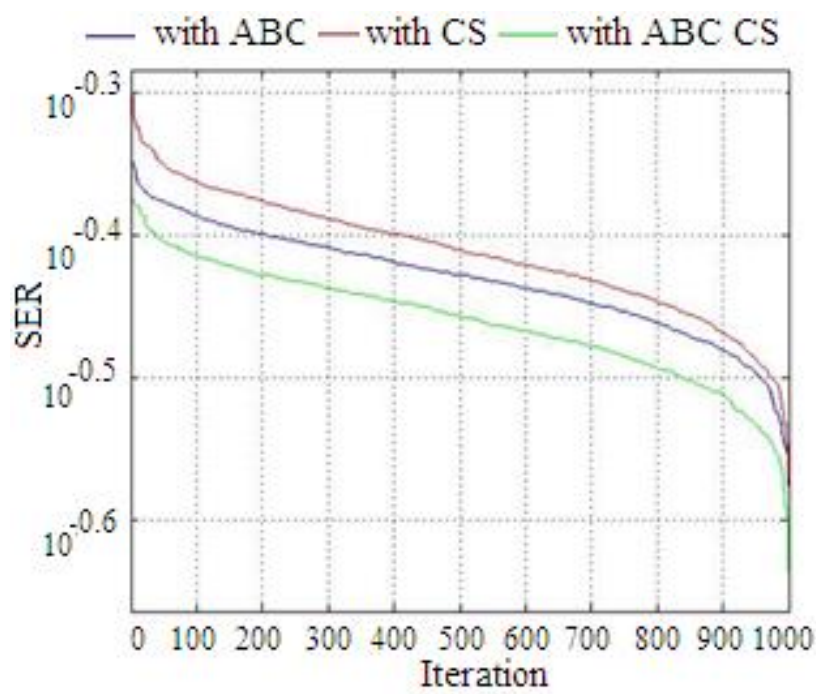

Figure.3 SER Comparison

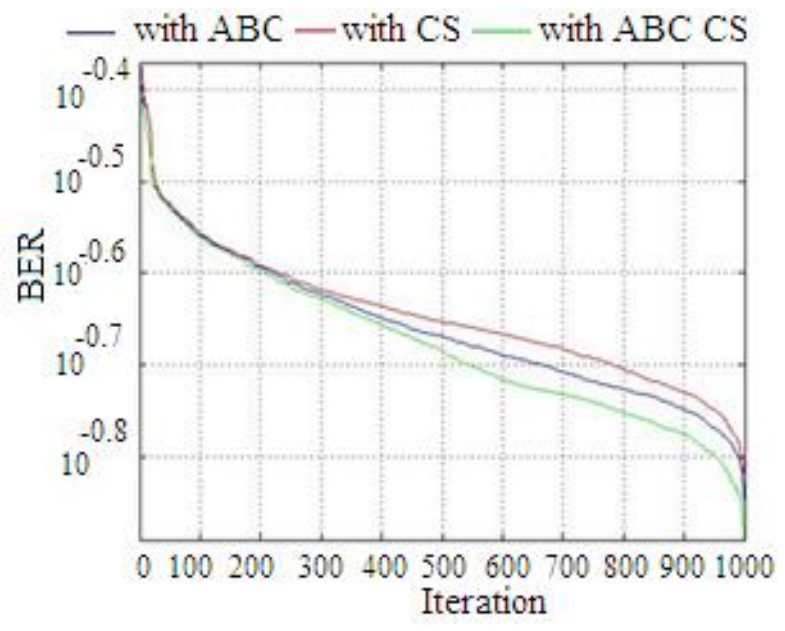

Figure.4 BER Comparison 


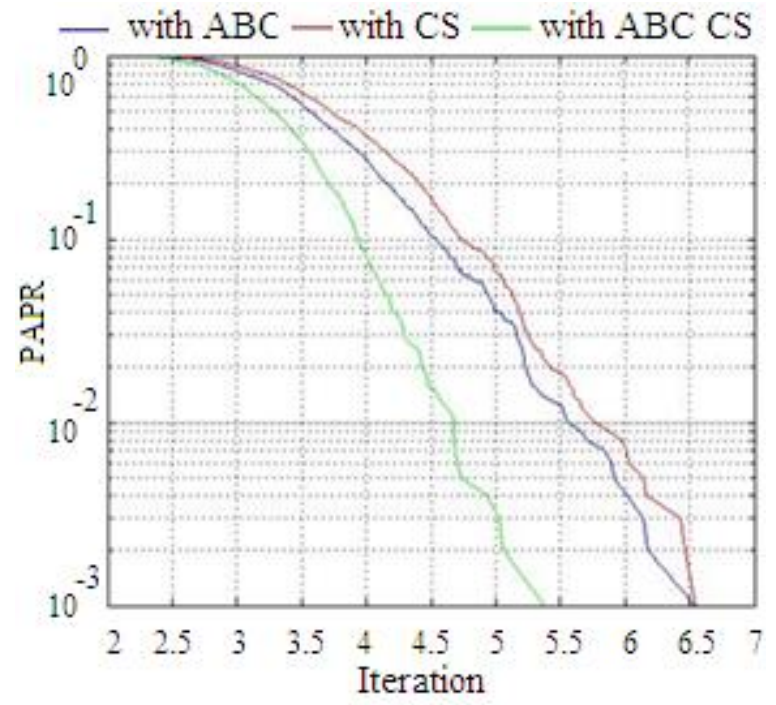

Figure.5 PAPR Comparison

The experiential CCDF is most much of the time used for surveying the PAPR. PAPR decrease capacity is controlled by the measure of CCDF lessening accomplished. CCDF offers a sign of the likelihood of the OFDM flag's envelope outperforming an evaluated PAPR edge inside the OFDM image and is given by,

$$
C C D F\left[\operatorname{PAPR}\left(g^{n}(t)\right)\right]=\operatorname{prob}\left[\operatorname{PAPR}\left(g^{n}(t)\right)>\delta\right](18)
$$

Where $g^{n}(t)$ - PAPR of the nth OFDM symbol

\section{$\delta$ - Threshold}

In figure 5, the simulation solutions of CCDF for plan are researched with unique improvement strategies. on the off chance that $\mathrm{CCDF}=100$ the , the PAPR expected by Cuckoo Search is $6.51 \mathrm{db}$ however by Artificial Bee Colony it is $6.5 \mathrm{db}$.The PAPR esteem accomplished by Artificial Bee Colony Cuckoo Search is 5.4db. Counterfeit Bee Colony Cuckoo Search can recognize ideal consequence of stage variable. It likewise diminishes the PAPR of OFDM flag proficiently. Accordingly we have seen that the PAPR decrease ability of proposed ABC-CS plan is great and SI is free contrasted with existing ABC, CS. Its computational multifaceted nature is additionally better contrasted with existing strategy.

\section{Conclusion}

The Partial Transmit Sequence (PTS) can be used in MIMO-OFDM plans to minimize the PAPR. A drawback of PTS is that, it needs numerous reverse quick Fourier/wavelet changes (IFFT/IDWT), thus achieving high computational adjusts in viable diagrams. In PTS conspire; the ideal collection of stage element esteem diminishes the PAPR. From now on, a versatile ABC improvement process is occupied with the anticipated strategy to choose the ideal stage consider esteem. The plan execution is evaluated inside the anticipated and the accessible frameworks to show the viability of the anticipated plan. As of now, the execution is surveyed for the sake of BER (Bit Error Rate), SER (Symbol Error Rate), and PAPR (Peak to Average Power Ratio) for the anticipated furthermore the accessible strategies, for example, $\mathrm{ABC}$ and $\mathrm{CS}$ calculations. In future by utilizing new methods we may diminish the PAPR in OFDM frameworks more.

\section{References}

[1]T. Jiang, C. Ni, C. Ye, Y. Wu and K. Luo, "A Novel Multi-Block Tone Reservation Scheme for PAPR Reduction in OQAM-OFDM Systems", IEEE Trans. on Broadcast., Vol. 61, No. 4, pp. 717-722, 2015.

[2] T. Jiang, W. Xiang, P. Richardson, J. Guo and G. Zhu, "PAPR Reduction of OFDM Signals Using Partial Transmit Sequences With Low Computational Complexity", IEEE Trans. on Broadcast., Vol. 53, No. 3, pp. 719-724, 2007.

[3]Y. Wang, W. Chen and C. Tellambura, "A PAPR Reduction Method Based on Artificial Bee Colony Algorithm for OFDM Signals", IEEE Transactions on Wireless Communications, Vol. 9, No. 10, pp. 2994-2999, 2010.

[4]H. Yoo, F. Guilloud and R. Pyndiah, "PAPR reduction for LDPC coded OFDM systems using binary masks and optimal LLR estimation", Signal Processing, Vol. 91, No. 11, pp. 2606-2614, 2011.

[5]J. Wang, J. Lee, J. Park, I. Song and Y. Kim, "Combining of Cyclically Delayed Signals: A Low-Complexity Scheme for PAPR Reduction in OFDM Systems", IEEE Trans. on Broadcast., Vol. 56, No. 4, pp. 577-583, 2010.

[6] J. Hmood, K. Noordin, H. Arof and S. Harun, "Peak-to-average power ratio reduction in alloptical orthogonal frequency division multiplexing system using rotated constellation approach", Optical Fiber Technology, Vol. 25, pp. 88-93, 2015.

[7]Y. Wang, J. Wang, K. Yi and B. Tian, "PAPR Reduction of OFDM Signals With Minimized EVM via Semidefinite Relaxation", IEEE Trans. Veh. Technol., Vol. 60, No. 9, pp. 4662-4667, 2011.

[8]P. Elavarasan and G. Nagarajan, "Peak-power reduction using improved partial transmit sequence in orthogonal frequency division 
multiplexing systems", Computers \& Electrical Engineering, Vol. 44, pp. 80-90, 2015.

[9]J. Chen, "Partial transmit sequences for PAPR reduction of OFDM signals with stochastic optimization techniques", IEEE Transactions on Consumer Electronics, Vol. 56, No. 3, pp. 12291234, 2010.

[10]M. F. Naeiny and F. Marvasti, "Selected Mapping Algorithm for PAPR Reduction of Space-Frequency Coded OFDM Systems without Side Information", IEEE Trans. Veh. Technol., Vol. 60, No. 3, pp. 1211-1216, 2011.

[11]L. Nadal, M. S. Moreolo, J. Fàbrega and G. Junyent, "Low complexity PAPR reduction techniques for clipping and quantization noise mitigation in direct-detection O-OFDM systems", Optical Fiber Technology, Vol. 20, No. 3, pp. 208-216, 2014.

[12]H. Chen, J. Yu, J. Xiao, Z. Cao, F. Li and L. Chen, "Nonlinear effect mitigation based on PAPR reduction using electronic pre-distortion technique in direct-detection optical OFDM system", Optical Fiber Technology, Vol. 19, No. 5, pp. 387-391, 2013.

[13]J. Zhou, Z. Zhang, T. Zhang, M. Guo, X. Tang, Z. Wang and Y. Qiao, "A combined PAPRreduction technique for asymmetrically clipped optical OFDM system", Optics Communications, Vol. 366, pp. 451-456, 2016.

[14]S. Shu, D. Qu, L. Li and T. Jiang, "Invertible Subset QC-LDPC Codes for PAPR Reduction of OFDM Signals", IEEE Trans. on Broadcast., Vol. 61, No. 2, pp. 290-298, 2015.

[15]K. Kim, "On the Shift Value Set of Cyclic Shifted Sequences for PAPR Reduction in OFDM Systems", IEEE Trans. on Broadcast., Vol. 62, No. 2, pp. 496-500, 2016.

[16]D. Qu, L. Li and T. Jiang, "Invertible Subset LDPC Code for PAPR Reduction in OFDM Systems with Low Complexity", IEEE Transactions on Wireless Communications, Vol. 13, No. 4, pp. 2204-2213, 2014. 